\title{
The Connor-Davidson Resilience Scale (CD-RISC): Testing the invariance of a uni-dimensional resilience measure that is independent of positive and negative affect
}

\author{
R.A. Burns*, K.J. Anstey \\ Ageing Research Unit, Centre for Mental Health Research, Australian National University, Canberra 0200, ACT, Australia
}

\section{A R T I C L E I N F O}

\section{Article history:}

Received 9 April 2009

Received in revised form 11 November 2009

Accepted 19 November 2009

Available online 12 January 2010

\section{Keywords:}

Resilience

Factor analysis

Well-being

Epidemiology

Psychometrics

\begin{abstract}
A B S T R A C T
Resilience comprises cognitive and behavioural tendencies that reflect dispositional character traits and patterns of behaviour that develop through life experience. Resilience is associated with positive mental and physical health outcomes although debate over its function as a predictor and/or outcome of successful stressful life conditions exists. Findings are confounded by a range of operational definitions. This study tested the factorial structure of the Connor and Davidson Resilience Scale (CD-RISC) and assessed its independence of two broad affective constructs, positive and negative affect. Participants $(n=1775)$ comprised the youngest adult cohort from the PATH study from Canberra, Australia. Results supported a uni-dimensional CD-RISC measure that was independent of affect at an item level, but supported strong associations between resilience and affect factors. Comparable Goodness of Fit Indices supported strict invariance between genders on an oblique 3-factor model of resilience and affect.
\end{abstract}

(c) 2009 Elsevier Ltd. All rights reserved.

\section{Introduction}

Resilience is a multi-dimensional construct that comprises a network of favourable attitudes and behaviours that enable adaptive coping strategies to acute and chronic stressful life events (Lamond et al., 2008; Rutter, 1985). Resilient people are typically characterised by internal locus of control, positive self-image and optimism (e.g. Cederblad, 1996; Werner \& Smith, 1992). These resilient and hardy characteristics are associated with better physical and mental health outcomes (Connor \& Davidson, 2003), more positive adaptive behaviours to negative life events (Aspinall \& MacNamara, 2005) and are protective against the onset of PostTraumatic Stress Disorder (King, King, Fairbank, Keane, \& Adams, 1998). In line with proponents of positive psychology (e.g. Ryan \& Deci, 2001), resilience is indicative of positive mental health (Maddi \& Khoshaba, 1994).

Despite the consensus over the characteristics and correlates of resilience, agreement relating to its temporal stability has been less unanimous. Evidence that purports a heritable component of resilience, as indicated by biological markers such as higher levels of dopamine, neuropeptide $Y$, testosterone, and increased functionality of 5- $\mathrm{HT}_{1 \mathrm{~A}}$ and benzodiazepine receptors (Charney, 2004; Gervai et al., 2005), supports temporal stability and suggests resilience incorporates a set of temporally-stable psychological resources

\footnotetext{
* Corresponding author. Tel.: +61 0261253132.

E-mail address: richard.burns@anu.edu.au (R.A. Burns).
}

available to the individual throughout the lifespan (Vaishnavi, Connor, \& Davidson, 2007). This is also supported by longitudinal survey data (e.g. Cederblad, 1996; Werner \& Smith, 1992). In contrast, notions of resilience as behavioural and cognitive responses to a specific event have been proffered (e.g. Lamond et al., 2008) and one's capacity for resilience appears shaped by age and life experience (e.g. Gillespie, Chaboyer, \& Wallis, in press). Consequently, resilience is described as either a set of heritable traits, an outcome of stressful life transactions, or as a process-construct reflecting an interaction between trait attitudes and behaviours with life experiences (Ahern, Kiehl, Sole, \& Byers, 2006).

Resilience measures commonly comprise self-report, have not been extensively validated, nor has their application been widely documented (Ahern et al., 2006). Yet, growing recognition of the impact of positive psychological states for individual and social well-being (Huppert, 2008), suggests an evaluation of available measures of resilience and their underlying factor structure and comparability at different stages of the life course, is needed. Similar positive well-being measures are now widely incorporated into national health surveys including the Health and Retirement Survey and the National Survey of Midlife Development (e.g. Ryff, Keyes, \& Hughes, 2004), and the German Socioeconomic Panel (e.g. Van Landeghem, 2008).

Well-being research typically delineates between related cognitive and affective psychological constructs (Ryan \& Deci, 2001). As a cognitive psychological resource, resilience may function to optimise subjective well-being by increasing positive and decreasing 
negative affectivity, but since measures of resilience frequently comprise self-report assessments, indices of resilience may simply reflect affect. However, Burns and Machin (2009) have differentiated between cognitive and affective well-being dimensions at the item level, whilst still moderately related at a higher-order factor level. It is important to determine a similar finding in respect to resilience and affect.

A number of resilience measures have been developed and include the Resilience Scale (RS; Wagnild \& Young, 1993), which assesses two factors, personal competence and acceptance of self and life; the Resilience Scale for Adults (RSA; Friborg, Hjemdal, Rosenvinge, \& Martinussen, 2003), which measures five factors: personal competence, social competence, family coherence, social support and personal structure; and the Connor-Davidson Resilience Scale (CD-RISC; Connor \& Davidson, 2003). The CD-RISC has been used with clinical and non-clinical populations (Connor \& Davidson, 2003), as a measure of psychological resources in moderating childhood maltreatment and adult psychiatric symptoms (Campbell-Sills, Cohan, \& Stein, 2006), and as an indicator for successful resilience training (Davidson et al. 2005). Only one large scale validation of the CD-RISC within a general population has been reported (Lamond et al., 2008).

The CD-RISC authors and others typically compute a total CDRISC score (e.g. Campbell-Sills et al., 2006; Lamond et al., 2008), yet post hoc analysis in the initial review (Connor \& Davidson, 2003) indicated a five-factor structure that reflected a multidimensional resilience scale. However, the methods employed in the initial report (Connor \& Davidson, 2003), and subsequent analyses (e.g. Campbell-Sills \& Stein, 2007; Vaishnavi et al., 2007) of the CD-RISC's psychometric properties, reveal several limitations to the proposed multi-dimensional nature of the CD-RISC. For example, Lamond et al. (2008) reported using a Principal Components extraction and orthogonal rotation method which is not appropriate for identifying a correlated factor structure (Burns \& Machin, 2009). Although using a Principal Axis Factoring method, Connor and Davidson (2003) also reported an orthogonal rotation method in their initial analysis which assumes that the resilience components are unrelated when a multi-dimensional resilience model should comprise oblique factors. Many of the initial factor loadings (Connor \& Davidson, 2003) revealed several moderately crossloading items and suggest that investigation of a more parsimonious structure is warranted. For example, Campbell-Sills and Stein (2007) used Exploratory Factor Analysis (EFA) with an oblique Promax rotation to identify a refined 10 -item uni-dimensional model.

\subsection{Aim}

Consequently, we seek to test the structural validity of the a priori five-factor model of the CD-RISC measure (Connor \& Davidson, 2003) in a large population-based sample of young-adults and subsequently test its independence from a measure of positive and negative affect.

\section{Method}

\subsection{Study design and participants}

The sample came from the PATH Through Life Project, a large community survey concerned with the health and well-being of randomly selected individuals from electoral rolls of Canberra or Queanbeyan, Australia, and where voting is compulsory (see Anstey, Dear, Christensen, \& Jorm, 2005). Results presented here concern the youngest cohort aged 20-24 years at wave 1 . Of 2404 commencing participants, 1978 were contactable at wave 3 in $2007 / 8$, when data on resilience were collected. As an exploratory investigation missing data was not imputed and only participants who provided full data $(N=1775 ; 45.9 \%=$ males $)$ for all measures were included. Participants had a mean education level of 15 years, and $88.3 \%$ rated their health as good, very good or excellent. Participants were mostly assessed in their homes and asked to complete a questionnaire under the supervision of a professional interviewer. Basic physical and cognitive tests were also carried out (e.g. blood pressure, grip strength, reaction time). Participants received a full description of the study and provided informed consent. The study was approved by the Human Research Ethics Committee at the Australian National University.

\subsection{Measures}

The Connor-Davidson Resilience Scale (CD-RISC; Connor \& Davidson, 2003) comprises 25 items that measure resilience or capacity to change and cope with adversity. Respondents indicated their response on a 5-point Likert scale with higher scores indicating greater resilience. The Positive and Negative Affect Schedule (PANAS; Watson, Clark, \& Tellegen, 1988) is a 20 item self-report measure of positive and negative affect (10 items per construct), that reflects both trait and state measures of affect, depending on the time reference included in the item operative. Individuals indicated their response on a 5-point Likert scale, with higher scores indicating greater well-being on that dimension. Due to an administrative oversight, two negative affect items were excluded from the questionnaire.

\subsection{Statistical analysis}

Confirmatory Factor Analysis (CFA) tested the a priori five-factor CD-RISC model (Connor \& Davidson, 2003) in AMOS v17 using Maximum Likelihood Estimation (MLE). Further model specification was undertaken with Exploratory Factor Analysis (EFA) in SPSS v17, using Principal Axis Factoring (PAF) and an oblique Direct Oblimin rotation (delta $=0$ ) since separate CD-RISC factors should reflect related resilience characteristics. We used Parallel Analysis (PA) to guide our EFA extraction. PA is a sample-based approach to estimating the number of factors and appears more highly robust than Kaiser's Rule or the Scree plot (Hayton, Allen, \& Scarpello, 2004). Goodness of Fit Indices (GFI) of the extracted EFA model were computed using AMOS v17 and compared to a priori five-factor model and a revised ten-item uni-dimensional model (Campbell-Sills \& Stein, 2007). CFA of the CD-RISC and PANAS tested the discriminant validity of the CD-RISC measure by evaluating the independence of resilience items from positive and negative affect. Factorial invariance between gender was tested using the Multi-Groups Function in AMOS v17.

\section{Results}

\subsection{Testing the structural validity of the RISC}

CFA of the a priori five-factor model (Connor \& Davidson, 2003) revealed a number of large Modification Indices (MI; >20) and items that did not discriminate the proposed factors. Several very high correlations indicate considerable overlap between four of the five latent variables (Table 1). In addition, GFI revealed poor fit (Table 2) for this model. Consequently, with debate over the extent to which the CD-RISC reflects a multidimensional (Connor \& Davidson, 2003) or uni-dimensional model (Campbell-Sills \& Stein, 2007), we used EFA to further explore the nature of the CDRISC. Results of a PA did not support an extraction of five factors, but indicated one factor with a raw eigenvalue greater than $95 \%$ of the PA, with three more eigenvalues greater than their corresponding 
Table 1

Correlations between factors of the a priori five CD-RISC model.

\begin{tabular}{llllll}
\hline & Factor 1 & Factor 2 & Factor 3 & Factor 4 & Factor 5 \\
\hline Factor 1 & 1 & .878 & .875 & .839 & .253 \\
Factor 2 & .878 & 1 & .901 & .718 & .217 \\
Factor 3 & .875 & .901 & 1 & .767 & .248 \\
Factor 4 & .839 & .718 & .767 & 1 & .275 \\
Factor 5 & .253 & .217 & .248 & .275 & 1 \\
\hline
\end{tabular}

mean PA eigenvalues. Therefore we used PAF in SPSS v17 to extract four factors, explaining $54 \%$ of the variance in the CD-RISC. However, most items loaded onto the first factor with one or two items cross-loading onto the other three factors, indicating that further refinement of the factor structure was warranted. Extracting three factors revealed considerable number of cross-loading items and shared variance between Factors 1 and $3\left(r^{2}=.609\right)$ whilst only items 3 and 9 loaded onto the second factor. With a criterion loading of $\leqslant .32$ (level at which a factor explains $10 \%$ unique item variance), a two-factor structure revealed several moderate crossloadings. Therefore a one-factor model was extracted, but both items 3 and 9 failed to load >.32 onto this factor. Re-running this model with items 3 and 9 deleted, revealed that all other items loaded onto this single factor, accounting for $40 \%$ of the explained variance, and supports Campbell-Sills and Stein's (2007) findings of a unitary resilience construct.

In comparison to the a priori five-factor model (Model 1) and our EFA derived uni-dimensional model (Model 2), GFI and the $\chi^{2}$ test of difference revealed that Model 3, an abridged 10-item version of the CD-RISC (Campbell-Sills \& Stein, 2007), reported better fit. However, GFI indicated that significant improvements were warranted for both the full-scale and shorter models and significant covariance paths between error terms were included for grossly large Modification Indexes $(\mathrm{MI} \geqslant 20)$. Considerable improvement in model fit was reported for all models, although comparable fit between the models was now reported. Preference for the uni-dimensional models was chosen on the grounds of parsimony (Table 2).

\subsection{Discriminant validity of the CD-RISC with affect between gender}

We extended our analyses to explore the relationship between the larger uni-dimensional CD-RISC model extracted from our EFA with a measure of affect, the PANAS (Watson et al., 1988). A CFA of the PANAS and CD-RISC items hypothesised a three-factor extraction excluding CD-RISC items 3 and 9 since these failed to load onto our earlier unitary factor. Based on our earlier criteria that items be excluded if they loaded $\geqslant .32$ on two or more factors, or failed to achieve this level on one factor, CD-RISC item 2 was also excluded from the analysis. All other item loadings and the lack of item misspecification confirmed the discrimination of PANAS and CD-RISC items and suggests that perceptions of resilience are independent of affect at the item level for the sample and by gender (Table 3 ). The CD-RISC was positively associated with positive affect $(r=.575, p<.001)$ whilst CD-RISC $(r=-.261, p<.001)$ and positive affect $(r=-.352, p<.001)$ were both negatively associated with negative affect. In comparison with Campbell-Sills and Stein's (2007) modified 10-item uni-variate model, not all of these ten items (identified in italics type in Table 3 ) were identified as the strongest loadings in this sample and this was consistent between gender.

Despite reporting a significant chi-square, other GFI revealed strong support for this three-factor model of resilience and affect $\left(\chi^{2}=2202.449, \quad \mathrm{df}=667, \quad p=.001 ; \quad\right.$ AGFI $=.926 ; \quad$ CFI $=.958 ;$ RMSEA $=.036$ (95\% CI: .034-.037)). Significant covariance paths between error terms were again included within factors that reported grossly large Modification Indexes $(\mathrm{MI} \geqslant 20)$. Although item loadings between gender were consistent (Table 3), multi-groups CFA tested for gender invariance (Table 4). Following Kline (2005), configural and metric invariance was supported with all factor loadings significant and equivalent between gender. Significant chisquare differences between an unconstrained model and three models that constrained, regression weights, error and variance terms failed to support strong and strict invariance, whilst GFI between models reported comparable moderate good fit of the data. In particular, a comparison of the RMSEA confidence intervals reveals fit for all models to be comparable across gender and suggests that this model is invariant.

\section{Discussion}

Our results failed to support the hypothesis that the CD-RISC comprises multiple resilience factors (e.g. Connor \& Davidson, 2003), but rather supported a one-factor CD-RISC model (Campbell-Sills \& Stein, 2007) comprising a revised 22-item CD-RISC scale. Published results (Connor \& Davidson, 2003) revealed that CD-RISC items 3 and 9 would be problematic in a uni-dimensional context since they reported cross-factor loadings and item-total scale correlations that were low. This was supported in this study suggesting that these items tap a latent construct different to the other CD-RISC items. The failure of CD-RISC item 2 to load onto the final solution was not surprising since its item-total scale correlation reported in Connor and Davidson's (2003) analysis was also comparatively low.

Table 2

A summary table of Goodness of Fit Indices from CFA of three models of the CD-RISC.

\begin{tabular}{|c|c|c|c|c|c|c|c|c|c|}
\hline & & $\chi^{2}$ & Df & $p$ & AGFI & CFI & RMSEA & AIC & Model comparisons $^{\mathrm{d}}$ \\
\hline Model $1^{\mathrm{a}}$ & Five-factor model & 2530.731 & 265 & .000 & .858 & .878 & $.069(.067-.072)$ & 2650.731 & $\mathrm{M} 1>\mathrm{M} 2$ \\
\hline Model $2^{\mathrm{b}}$ & Uni-dimensional model & 2874.717 & 230 & .000 & .826 & .852 & $.081(.078-.083)$ & 2966.717 & \\
\hline Model $3^{c}$ & 10-Item uni-dimensional model & 200.105 & 35 & .000 & .963 & .972 & $.052(.045-.059)$ & 240.105 & $\begin{array}{l}\text { M3 > M1 } \\
\text { M3 > M2 }\end{array}$ \\
\hline Model $4^{\mathrm{a}}$ & Model 1 with significant covariance paths & 154.866 & 144 & .253 & .984 & .999 & $.007(.000-.013)$ & 516.866 & $\mathrm{M} 4=\mathrm{M} 3$ \\
\hline Model $5^{\text {b }}$ & Model 2 with significant covariance paths & 155.385 & 127 & .044 & .984 & .998 & $.011(.002-.017)$ & 458.358 & $\begin{array}{l}\mathrm{M} 5=\mathrm{M} 3 \\
\mathrm{M} 5=\mathrm{M} 4\end{array}$ \\
\hline Model $6^{\mathrm{c}}$ & Model 3 with significant covariance paths & 26.646 & 21 & .183 & .992 & .999 & $.012(.000-.025)$ & 94.646 & $\begin{array}{l}M 6>M 3 \\
M 6=M 4 \\
M 6=M 5\end{array}$ \\
\hline
\end{tabular}

${ }^{a}$ Model 1 and 4: a priori five-factor model (Connor and Davidson, 2003).

b Model 2 and 5: uni-dimensional model of our EFA derived model.

c Model 3 and 6: 10-item uni-dimensional model (Campbell-Sills and Stein, 2007).

d Comparisons of model fit undertaken with $\chi^{2}$ test of difference $(p<.05)$ : $(>)$ indicates better fitting model; $(<)$ indicates worse fitting model; $(=)$ indicates models fit comparatively. 
Table 3

Item loadings from a CFA analysis of the CD-RISC and PANAS items.

\begin{tabular}{|c|c|c|c|c|c|c|c|c|c|}
\hline \multirow[b]{2}{*}{ RISC items } & \multicolumn{3}{|c|}{ Whole sample } & \multicolumn{3}{|l|}{ Males } & \multicolumn{3}{|l|}{ Females } \\
\hline & CD-RISC & NA & PA & CD-RISC & NA & PA & CD-RISC & NA & PA \\
\hline 17 & .750 & -.032 & .026 & .737 & -.100 & -.005 & .752 & .021 & .057 \\
\hline 24 & .723 & .065 & .066 & .721 & .092 & -.080 & .721 & .047 & .057 \\
\hline 16 & .685 & -.004 & .035 & .718 & .025 & -.034 & .659 & -.008 & .041 \\
\hline 4 & .684 & -.064 & -.017 & .697 & -.085 & .055 & .669 & -.040 & .023 \\
\hline 12 & .678 & .036 & .049 & .691 & .093 & -.054 & .672 & -.020 & .037 \\
\hline 5 & .672 & -.022 & .043 & .692 & -.023 & -.023 & .651 & -.023 & .063 \\
\hline 11 & .663 & -.010 & .105 & .680 & .005 & -.089 & .650 & -.025 & .113 \\
\hline 23 & .647 & -.022 & .079 & .599 & -.054 & -.086 & .678 & .013 & .087 \\
\hline 18 & .618 & .016 & -.120 & .640 & .053 & .109 & .603 & .012 & -.129 \\
\hline 14 & .617 & -.111 & -.042 & .632 & -.143 & .081 & .603 & -.071 & -.005 \\
\hline 8 & .611 & -.064 & .027 & .582 & -.060 & -.051 & .625 & -.070 & .016 \\
\hline 15 & .608 & .035 & -.008 & .592 & .025 & .006 & .617 & .057 & -.003 \\
\hline 19 & .607 & -.055 & -.058 & .561 & -.062 & .007 & .635 & -.043 & -.089 \\
\hline 7 & .585 & .040 & -.040 & .563 & .026 & .022 & .597 & .045 & -.044 \\
\hline 21 & .532 & .012 & .202 & .514 & -.014 & -.200 & .555 & .011 & .206 \\
\hline 22 & .523 & -.194 & .200 & .521 & -.207 & -.190 & .524 & -.194 & .210 \\
\hline 10 & .476 & .019 & .057 & .506 & .026 & -.070 & .474 & -.016 & .036 \\
\hline 6 & .473 & -.025 & -.012 & .493 & -.062 & .020 & .443 & .015 & .008 \\
\hline 1 & .468 & -.097 & -.002 & .424 & -.100 & -.015 & .501 & -.096 & -.013 \\
\hline 25 & .464 & .003 & .179 & .423 & .010 & -.211 & .500 & -.019 & .148 \\
\hline 20 & .459 & .091 & -.052 & .451 & .097 & .040 & .467 & .075 & -.061 \\
\hline 13 & .362 & -.057 & .124 & .370 & -.091 & -.181 & .390 & -.086 & .070 \\
\hline \multicolumn{10}{|l|}{ PANAS } \\
\hline Scared & -.002 & .785 & .026 & -.007 & .771 & -.042 & .002 & .789 & .016 \\
\hline Nervous & -.054 & .741 & .100 & -.071 & .667 & -.102 & -.051 & .792 & .108 \\
\hline Afraid & .009 & .737 & .017 & .005 & .691 & -.005 & .011 & .764 & .028 \\
\hline Distressed & .037 & .709 & -.155 & .002 & .767 & .121 & .065 & .662 & -.185 \\
\hline Guilty & -.039 & .666 & .001 & -.035 & 649 & .037 & -.039 & .680 & .028 \\
\hline Jittery & -.022 & .658 & .069 & -.020 & 652 & -.081 & -.028 & .664 & .057 \\
\hline Upset & .045 & .649 & -.195 & .048 & .652 & .195 & .050 & .638 & -.204 \\
\hline Ashamed & -.058 & .645 & -.030 & -.015 & .707 & .046 & -.089 & .613 & -.026 \\
\hline Enthusiastic & -.003 & .034 & .873 & -.011 & .033 & -.856 & .010 & .035 & .879 \\
\hline Interested & .028 & .005 & .830 & .044 & -.012 & -.793 & .025 & .015 & .854 \\
\hline Inspired & .041 & .004 & .749 & .036 & .016 & -.748 & .046 & -.005 & .748 \\
\hline Excited & -.037 & .048 & .736 & -.041 & .041 & -.749 & -.030 & .045 & .721 \\
\hline Determined & .138 & .149 & .724 & .134 & .144 & -.700 & .147 & .153 & .737 \\
\hline Strong & .045 & -.140 & .670 & .052 & -.117 & -.644 & .040 & -.141 & .698 \\
\hline Proud & .030 & -.021 & .644 & .058 & -.007 & -.673 & .007 & -.037 & .618 \\
\hline Alert & .047 & -.020 & 619 & .065 & -.046 & -.581 & .034 & .012 & .654 \\
\hline Attentive & -.007 & -.084 & .618 & -.008 & -.046 & -.578 & .008 & -.115 & .643 \\
\hline Active & .003 & -.093 & .613 & -.038 & -.076 & -.674 & .022 & -.099 & .580 \\
\hline
\end{tabular}

Bold and italics type indicate the 10-items identified in Campbell-Sills and Stein's (2007) analyses.

Table 4

Multi-groups analysis of a three-factor model of CD-RISC and PANAS testing for invariance between gender.

\begin{tabular}{|c|c|c|c|c|c|c|c|}
\hline & $\chi^{2}$ & df & $p$ & AGFI & CFI & RMSEA & $\chi^{2}$.diff. test \\
\hline Unconstrained model & 2950.936 & 1354 & .000 & .905 & .957 & $.026(.025-.027)$ & - \\
\hline Constrained measurement weights & 3040.188 & 1391 & .000 & .905 & .955 & $.026(.025-.027)$ & $p<.001$ \\
\hline Constrained structural co-variances & 3057.363 & 1397 & .000 & .905 & .955 & $.026(.025-.027)$ & $p<.001$ \\
\hline Constrained measurement residuals & 3289.363 & 1497 & .000 & .905 & .952 & $.026(.025-.027)$ & $p<.001$ \\
\hline
\end{tabular}

$\chi^{2}$ diff. test computed with the unconstrained model as the reference model.

Support for our uni-variate CD-RISC model is reflected in the reported GFI and that most of the explained variance was accounted for by the first factor. Our revised 22-item model indicated CDRISC's independence of two broad affect states, with moderate coefficients between affective and cognitive well-being components reported. In comparison to Campbell-Sills and Stein's (2007) abridged 10-item CD-RISC model, the longer uni-dimensional model performed comparatively. Importantly, examination of the item loadings in our EFA highlighted the limitation of using an abridged version of the CD-RISC as the 10 -items reported in Campbell-Sills and Stein's (2007) analyses were not the strongest loading items in our representative community sample. However, whilst other manifest items were more indicative of the resilience latent construct in our sample, a strong association $(r=.950)$ between the factor scores of the longer and shorter scale forms suggests that these differences are not substantial and that the two scale forms are comparable. We recognise that a shorter item pool is more time efficient and user-friendly within the context of a larger battery of survey questionnaires. Further factorial analysis of all the CD-RISC items may yet indicate consistency in those items that are identified as common indicators of a uni-dimensional model of resilience for a short scale format.

Resilience may reflect a multi-dimensional structure of self attitudes. As with other models of self-referent beliefs (e.g. Shavelson, Hubner, \& Stanton, 1976) generalised resilience may reflect a set of latent constructs that comprise specific resilience components 
which relate to an individual's array of coping strategies, self-efficacy beliefs and perceived degree of self-determination. Consequently, measurement of context-specific resilience beliefs may better indicate the role of resilience in providing a buffer against the impact of life events. This is clearly an area that needs further refinement as is delineating the role of resilience as an outcome or predictive construct, however the CD-RISC does not assess such a model. Importantly though, the CD-RISC is independent of affect at an item level and supports previous findings relating to the related yet distinctive nature of cognitive and affective components of well-being (Burns \& Machin, 2009).

A number of limitations are identified. The CD-RISC scale was only introduced in the third wave of the PATH study and to date, data collection has only been completed for the younger adult cohort. It is important to test the validity of these findings with the other age cohorts as data become available. Furthermore, participants were relatively highly educated and ethnically non-diverse and so the findings need to be evaluated in more diverse populations. Age effects and other sampling characteristics may influence item response which can be reflected in item loadings in EFA. However, this is unlikely to impact on these findings since participants were similar in age. In fact, analysis of invariance of the CD-RISC and the PANAS revealed consistency in factor structure between gender and supported strict invariance. Although the results of this study are more appropriate for generalisation to the Australian population, a further strength is that participants were drawn from the electoral roll, unlike other analyses (e.g. Campbell-Sills \& Stein, 2007) which comprise convenience and clinical samples.

\section{Conclusion}

This study supported the uni-dimensional structure of the CDRISC and differentiated the CD-RISC items with affect whilst a strong relationship between these constructs at a higher-order level was reported. Future research should identify either a causal or reciprocal relationship between resilience and affect. Also, the independence of resilience with other cognitive well-being constructs, including mastery, has received little empirical substantiation.

\section{Acknowledgements}

We thank Trish Jacomb, Karen Maxwell, Helen Christensen, Andrew MacKinnon, Peter Butterworth and Simon Easteal and the PATH interviewers. Funding was provided by NHMRC grant \# 418039.

\section{References}

Ahern, N. R., Kiehl, E. M., Sole, M. L., \& Byers, J. (2006). A review of instruments measuring resilience. Issues in Comprehensive Paediatric Nursing, 29, 103-125.

Anstey, K. J., Dear, K., Christensen, H., \& Jorm, A. F. (2005). Biomarkers, health, lifestyle and demographic variables as correlates of reaction time performance in early, middle, and late adulthood. Quarterly Journal of Experimenta Psychology, 58A, 5-21.

Aspinall, L. G., \& MacNamara, A. (2005). Taking positive changes seriously. Cancer $104,2549-2556$
Burns, R. A., \& Machin, M. A. (2009). Investigating the structural validity of Ryff's psychological well-being scales across two samples. Social Indicators Research, 93(2), 359-375.

Campbell-Sills, L., Cohan, S. L., \& Stein, M. B. (2006). Relationship of resilience to personality, coping, and psychiatric symptoms in young adults. Behaviour Research and Therapy, 44, 585-599.

Campbell-Sills, L., \& Stein, M. B. (2007). Psychometric analysis and refinement of the Connor-Davidson Resilience Scale (CD-RISC): Validation of a 10-item measure of resilience. Journal of Traumatic Stress, 20(6), 1019-1028.

Cederblad, M. (1996). Fifty years of epidemiologic studies in child and adolescent psychiatry in Sweden. Nordic Journal of Psychiatry, 50, 55-66.

Charney, D. S. (2004). Psychobiological mechanisms of resilience and vulnerability: Implications for successful adaptation to stress. American Journal of Psychiatry, $161,195-216$.

Connor, K. M., \& Davidson, J. R. T. (2003). Development of a new resilience scale: The Connor-Davidson Resilience Scale (CD-RISC). Depression and Anxiety, 18, 76-82.

Davidson, J. R., Payne, V. M., Connor, K. M., Foa, E. B., Rothbaum, B. O., Hertzberg, M. A., et al. (2005). Trauma, resilience, and saliostatis: Effects of treatment on posttraumatic stress disorder. International Clinical Psychopharmacology, 20, 43-48.

Friborg, O., Hjemdal, O., Rosenvinge, J. H., \& Martinussen, M. (2003). A new rating scale for adult resilience. What are the central protective resources behind healthy adjustment? International Journal of Methods in Psychiatric Research, 12 65-76.

Gervai, J., Nemoda, Z., Lakatos, K., Ronai, Z., Toth, I., Ney, K., et al. (2005). Transmission disequilibrium tests confirm the link between DRD4 gene polymorphism and infant attachment. American Journal of Medical Genetics Part B (Neuropsychiatric Genetics), 132B, 126-130.

Gillespie, B.M., Chaboyer, W., \& Wallis, M. (In press). The influence of personal characteristics on the resilience of operating room nurses: A predictor study. International Journal of Nursing Studies, doi: 10.1016/j.ijnurstu.2007.08.006.

Hayton, J. C., Allen, D. G., \& Scarpello, V. G. (2004). Factor retention decisions in exploratory factor analysis: A tutorial on parallel analysis. Organizational Research Methods, 7(2), 191-205.

Huppert, F. (2008) Psychological Wellbeing: Evidence regarding its causes and consequences - State-of-Science Review: SR-X2, Government Office for Science Foresight Project, Mental Capital and Wellbeing: Making the most of ourselves in the 21st Century, London.

King, L. A., King, D. W., Fairbank, J. A., Keane, T. M., \& Adams, G. A. (1998). Resiliencerecovery factors in posttraumatic stress disorder among female and male Vietnam veterans: Hardiness, postwar social support, and additional stressful life events. Journal of Personality and Social Psychology, 74, 420-434.

Kline, R. B. (2005). Principles and practice of structural equation modeling (2nd ed.). New York: Guilford Press.

Lamond, A. J., Depp, C. A., Allison, M., Langer, R., Reichstadt, J., Moore, D. J., et al. (2008). Measurement and predictors of resilience among community-dwelling older women. Journal of Psychiatric Research, 43(2), 148-154.

Maddi, S. R., \& Khoshaba, D. M. (1994). Hardiness and mental health. Journal of Personality Assessment, 63, 265-274.

Rutter, M. (1985). Resilience in the face of adversity: Protective factors and resistance to psychiatric disorders. British Journal of Psychiatry, 25, 173-180.

Ryan, R., \& Deci, E. L. (2001). On happiness and human potentials: A review of research on hedonic and eudaimonic well-being. Annual Review of Psychology, $52,141-166$.

Ryff, C. D., Keyes, C. L. M., \& Hughes, D. L. (2004). Psychological well-being in MIDUS: Profiles of ethnic/racial diversity and life course uniformity. In O. G. Brim, C. D. Ryff, \& R. C. Kessler (Eds.), How healthy are we? A national study of well-being at midlife (pp. 398-422). Chicago: University of Chicago Press.

Shavelson, R., Hubner, J., \& Stanton, G. (1976). Self concept: Validation of construct interpretations. Review of Educational Research, 46, 407-441.

Vaishnavi, S., Connor, K., \& Davidson, J. R. T. (2007). An abbreviated version of the Connor-Davidson Resilience Scale (CD-RISC), the CD-RISC2: Psychometric properties and applications in psychopharmacological trials. Psychiatry Research, 152, 293-297.

van Landeghem, B.G.M. (2008) Human Well-Being over the Life Cycle: Longitudinal Evidence from a 20-Year Panel. LICOS Discussion Papers 21308, LICOS - Centre for Institutions and Economic Performance, K.U. Leuven.

Wagnild, G. M., \& Young, H. M. (1993). Development and psychometric validation of the resilience scale. Journal of Nursing Measurement, 1, 165-178.

Watson, D., Clark, A., \& Tellegen, A. (1988). Development and validation of brief measures of positive and negative affect: The PANAS scales. Journal of Personality and Social Psychology, 54, 1063-1070.

Werner, E. E., \& Smith, R. S. (1992). Overcoming the odds. High risk children from birth to adulthood. London: Cornell University Press. 